\title{
Analysis of the Carbohydrate Composition of Axonally Transported Glycoconjugates in Sciatic Nerve
}

\author{
Clyde E. Hart and John G. Wood \\ Department of Anatomy, Emory University School of Medicine, Atlanta, Georgia 30322
}

Glycosidase enzyme digestion in combination with postembedding lectin cytochemistry was used to study the carbohydrate composition of axonally transported glycoproteins. A cold block procedure for the interruption of axonal transport was employed to increase selectively the population of anterograde moving components on the proximal side of the transport block. Electron-microscopic observations revealed that a cold block applied to the sciatic nerve of an anesthetized rat produced an increase in axonal smooth membrane vesicles at a site directly proximal to the cold block. Postembedding lectin cytochemistry of the sciatic nerve demonstrated a substantial increase in concanavalin $A$ (Con A), wheat germ agglutinin (WGA), and succinylated WGA binding sites in axons directly proximal to the cold block. Endoglycosidase $H$ (endo $H$ ) digestion prior to lectin cytochemistry characterized a large population of the axonally transported Con $\mathrm{A}$ binding sites as polymannose and/or hybrid $\mathrm{N}$-linked oligosaccharides (endo $\mathrm{H}$-susceptible). A distinct population of neuraminidase-resistant WGA binding sites was also found in axons directly proximal to the transport block. The concomitant increase in smooth membrane vesicles and lectin binding sites in axons at the transport block supports the hypothesis that a system(s) of smooth membrane inside the axon is involved in the transport of glycoproteins from the cell soma to their cell surface destinations. Results of glycosidase digestions and lectin cytochemistry experiments suggest that many of the axonally transported glycoprotein carbohydrates are polymannose and/or hybrid $\mathrm{N}$-linked oligosaccharides. This observation is especially interesting in relation to our previous reports, which indicated that most lectin binding sites on the neuronal cell surface are composed of complex oligosaccharides.

Lectin cytochemistry in conjunction with glycosidase enzyme digestions has been used in our laboratory to study the relationships between intra- and extracellular glycoconjugates in nervous tissue (Hart and Wood, 1984, 1985; Wood and McLaughlin, 1976; Wood et al., 1974, 1981). The results of these studies show that the cisternae of membranous organelles in the somata, dendrites, axons, and presynaptic terminals of cerebellar neurons contain a majority of lectin binding sites that are distinct from the lectin binding sites of the neuronal plasma membrane as determined by differences in their susceptibility to endo- and exo-glycosidase enzyme digestions. At least some of these lectin-labeled membrane compartments are the sites of protein translation (rough endoplasmic reticulum, or RER), posttranslational modification (Golgi complex), and perhaps the vehicle for transport to the synaptic terminal cell surface (see Grafstein and Forman, 1980; Hammerschlag, 1984; Hammer-

\footnotetext{
Received Mar. 18, 1985; revised Sept. 30, 1985; accepted Oct. 30, 1985.

We wish to thank Antonio Aguilar for help with the photomicrographs and Janet Vogt for typing the manuscript. This work was supported by USPHS Grant NS-1773I.

Correspondence should be addressed to Dr. Wood at the above address.

Copyright (c) 1986 Society for Neuroscience $0270-6474 / 86 / 051365-07 \$ 02.00 / 0$
}

schlag and Stone, 1982, for reviews). Therefore, the ability to characterize in situ the carbohydrate composition of lectin binding sites of intracellular membrane compartments and on the cell surface may further our understanding of the site(s) of glycoprotein oligosaccharide processing in neurons.

In this study we have used a modification of the cold block technique of Hanson (1978) to interrupt axonal transport in the sciatic nerve of rats in order to increase the content of anterogradely transported glycoproteins at a focal point along the nerve. Results of postembedding lectin cytochemistry and endo- and exo-glycosidase digestions of the cold-blocked sciatic nerve indicatcd that some of the axonally transported glycoprotein carbohydrates were polymannose or hybrid oligosaccharides. A portion of this work has appeared in abstract form (Hart and Wood, 1983).

\section{Materials and Methods}

\section{Materials}

Concanavalin A (Type IV), $\alpha$-methyl-D-mannoside, HRP (Type VI), 3-3' - diaminobenzidine, protease (Type VI, from Streptomyces griseus), and neuraminidase (Type VI, from Clostridium perfringens) were from Sigma Chemical Company (St. Louis, MO); endo- $\beta$ - $N$-acetylglucosaminidase $\mathrm{H}$ was from Miles Laboratories, Inc. (Elkhart, IN); fluorescein isothiocyanate (FITC)-labeled lectins were from EY Laboratories Inc. (San Mateo, CA); and glutaraldehyde ( $8 \%$ ) and paraformaldehyde from Polysciences Inc. (Warrington, PA). All other chemicals were reagent grade. Adult Sprague-Dawley rats (male), 1-2 months old, were used for this study.

\section{Endo $H$ digestion of hybrid and complex glycoproteins in vitro}

The lectin binding characteristics of specific glycoproteins with known $\mathrm{N}$-linked oligosaccharide compositions were studied using ovalbumin (polymannose and hybrid ligosaccharides) and fetuin (complex oligosaccharides). Each protein was dissolved in distilled water with 1.2 -fold weight excess SDS and boiled for $5 \mathrm{~min}$. The protein solutions were then dialyzed for $48 \mathrm{hr}$ in a total of 3 liters of distilled water containing $0.15 \%$ SDS. Protein concentrations were determined by the Lowry procedure (Lowry et al., 1951). Ovalbumin $(0.5 \mathrm{mg})$ and fetuin $(0.5 \mathrm{mg})$ were each digested with endo $\mathrm{H}(7.5 \mathrm{mU})$ in $0.1 \mathrm{M}$ citrate-phosphate buffer ( $\mathrm{pH} \mathrm{5.6)}$ with $1 \mathrm{~mm}$ phenylmethylsulfonyl fluoride (PMSF) for $20 \mathrm{hr}$. The enzyme digestions were stopped by the addition of an equal volume of $0.5 \mathrm{M}$ Tris (pH 6.8) containing $2 \%$ SDS, $30 \%$ glycerol, and $0.25 \mathrm{~m}$ DTT.

The action of endo $\mathrm{H}$ on the glycoproteins was assayed by three techniques: (1) the difference in migration distances between control and cnzymc-treatcd proteins on $12 \%$ polyacrylamide gels stained with Coomassie blue using the discontinuous buffer system of Laemmli (1970); (2) lectin staining of the gel by the procedure of Wood and Sarinana (1975); or (3) electroblotting the gel onto nitrocellulose by the method of Towbin et al. (1979) and lectin staining the nitrocellulose transblot essentially by the steps described by Wood and Sarinana (1975), except for a preincubation step for $1 \mathrm{hr}$ at room temperature with $3 \%$ BSA in PBS and the addition of 3\% BSA to the lectin incubation and HRP steps. 


\section{Axonal transport blocks}

For these studies the animal was anesthetized with Nembutal $(1 \mathrm{cc} / \mathrm{kg}$ body weight), and the hair was removed from the lateral hindquarters. The sciatic nerve beneath the gluteal muscles was exposed and carefully dissected free of most connective tissue.

A modification of the cold block technique of Hanson (1978) was used to interrupt axonal transport in the sciatic nerve of rats. To cool the nerve a hook-shaped sterling silver wire $(0.5 \mathrm{~mm}$ diameter) attached to the bottom of a double-walled brass cylinder was carefully placed around the exposed sciatic nerve of an anesthetized rat. A chilled solution of propylene glycol and water (1:1) was circulated through the double-walled cylinder to produce a temperature at the silver wire of $0-5^{\circ} \mathrm{C}$. At a point $3 \mathrm{~mm}$ proximal to the chilled silver wire, an identical device was placed around the sciatic nerve, and warm water was circulatcd through the cylinder to produce a temperature of $37^{\circ} \mathrm{C}$ at the wire. Warming of the nerve proximal to the cold block was necessary to produce a focal point of inhibition of axonal transport as described previously (Tsukita and Ishikawa, 1979, 1980). After a 6 hr cold block, the positions of the silver wires were marked on the adjacent muscle with India ink and the animal was fixed by vascular perfusion as described previously (Hart and Wood, 1984).

Control experiments were performed in parallel with the same animal by placing silver wires, at ambient temperature, around the opposite sciatic nerve. These wires were left in place for the same period of time as the experimental cold block wires. The control and experimental sciatic nerves were processed further in an identical fashion. After a 20 min perfusion with fixative, both sciatic nerves were flooded with fixative for another $20 \mathrm{~min}$. During this time the nerves were cut both proximal and distal to the cold block in a way to identify the different ends of the cut nerve and carefully dissected out of the animal. The nerves were then placed in fixative for the remainder of a period of time to total $60 \mathrm{~min}$ beginning from the start of the perfusion. The nerve segments were then placed in $4 \%$ paraformaldehyde in $0.12 \mathrm{M}$ Millonig's buffer (Millonig, 1961) overnight at $4^{\circ} \mathrm{C}$. The next morning the nerves were washed in PBS, sectioned longitudinally at $200 \mu \mathrm{m}$ on a Lancer vibratome, and processed for postembedding lectin cytochemistry or electron microscopy.

\section{Embedment and etching of tissue}

The primary fixative and embedding procedure used in prcvious reports of pre-embedding lectin cytochemistry (Hart and Wood, 1984; Wood et al., 1974, 1981) was used for postembedding techniques except for the $\mathrm{OsO}_{4}$ postfixation step. The tissue embedded in Embed-Araldite was sectioned at $0.5 \mu \mathrm{m}$ and mounted on glass microscope slides. The section plastic was removed by etching with a solution of saturated sodium ethoxide: absolute ethanol (1:4) as described by Lane and Europa (1965). The etched tissue sections were rinsed in absolute ethanol, hydrated, and rinsed with buffer in preparation for enzyme digestion and lectin labeling. In some experiments a protease ( $S$. griseus) digestion $(0.1 \%)$ in $\mathrm{PBS}$ at $37^{\circ} \mathrm{C}$ prior to lectin cytochemistry was performed to confirm that the lectin receptors in the etched tissue sections were glycoproteins.

\section{Characterization of Con $A$ binding sites}

Endo $\mathrm{H}$ (0.1 unit) was dissolved in $200 \mu \mathrm{l} 0.10 \mathrm{M}$ citrate-phosphate buffer (pH 5.6). In some experiments, $0.05 \%$ SDS was included in the enzyme incubation. The final solution contained $1.0 \mathrm{~mm}$ phenylmethylsulfonyl fluoride (Sigma) as a protease inhibitor. The etched tissue sections were digested with the endo $\mathrm{H}$ solution in a moist environment at $37^{\circ} \mathrm{C}$ for $20 \mathrm{hr}$. Etched tissue sections incubated in $200 \mu \mathrm{l}$ of $0.10 \mathrm{M}$ citrate-phosphate buffer minus endo $\mathrm{H}$ were used for the enzyme control.

After cndo $\mathrm{H}$ digestion, or an incubation in buffer without enzyme, the sections were washed for $10 \mathrm{~min}$ with citrate-phosphate buffer (two changes) followed by a PBS wash for $15 \mathrm{~min}$ (three changes). The tissue sections were then processed for Con A lectin cytochemical labeling as described previously for pre-embedding experiments (Hart and Wood, 1984). A slide of etched tissue sections in the citrate-phosphate buffer minus endo $\mathrm{H}$ was incubated with Con A in PBS containing $0.2 \mathrm{M}$ $\alpha$-methyl-D-mannoside as a hapten control. After the diaminobenzidine incubation step, the sections were washed for $30 \mathrm{~min}$ PBS (three changes) and mounted with $20 \%$ glycerol in PBS.

\section{Characterization of $W G A$ binding sites}

Neuraminidase enzyme digestion (1.0 unit, from $C$. perfringens) of etched tissue sections was performed in $250 \mu \mathrm{l}$ of $0.15 \mathrm{M}$ sodium acetate

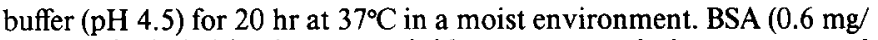
$\mathrm{ml}$ ) was included in the neuraminidase enzyme solution as suggested by Cassidy et al. (1965) to prevent the progressive inactivation of the enzyme during long incubation periods. After enzyme digestion of the tissue sections, the slides were rinsed in sodium acetate buffer $(15 \mathrm{~min})$ and subsequently in PBS (15 min, three changes) prior to lectin cytochemistry.

The enzyme-treated and control tissue sections (incubated in buffer minus the enzyme) were preincubated with $0.5 \%$ BSA in PBS prior to and during lectin cytochemistry to inhibit nonspccific binding of the lectins to the tissue sections. The sections were incubated with either WGA-FITC $(0.25 \mathrm{mg} / \mathrm{ml}$ in PBS) or succinylated WGA (sWGA)-FITC $\left(0.2 \mathrm{mg} / \mathrm{ml}\right.$ in PBS) for $3 \mathrm{hr}$ at $37^{\circ} \mathrm{C}$ in a moist environment. The native lectin and its succinylated derivative were used in combination with neuraminidase digestion to help identify populations of carbohydrates that contained both internal G1 cNAc and terminal NANA sugars (binds WGA and not sWGA) or oligosaccharides that contain GlcNAc without terminal NANA sugars (binds WGA and sWGA) (Bhavanandan and Katlic, 1979; Monsigny et al., 1980). Lectin control tissue sections were treated exactly the same as enzyme control tissue sections, except that the appropriate hapten sugar (200 mM GIcNAc for WGA and sWGA) was included in the lectin incubation step. Following the lectin incubations, the tissue sections were washed in PBS ( $25 \mathrm{~min}$, three changes) and mounted with $20 \%$ glycerol in PBS.

\section{Photomicrography}

The lectin-FITC-labeled tissue sections were photographed with a Leitz Laborlux 12 microscope using an epifluorescence illumination system. Peroxidase-labeled tissue was observed and photographed using phasecontrast optics. To analyze the effects of enzyme treatment on lectin labeling of the tissue sections, identical exposure conditions were utilized for both control and experimental tissue sections.

\section{Results}

\section{Endo $H$ digestion of ovalbumin and fetuin}

Endo $\mathrm{H}$ digestion of glycoproteins with known carbohydrate compositions was designed to prove that removal of specific glycoprotein oligosaccharides resulted in a loss of spccific lectin binding capabilities. Ovalbumin, a highly glycosylated protein with a mixture of high mannose and hybrid N-linked oligosaccharides (Kobata, 1979) lost the ability to bind Con A after an incubation with endo $\mathrm{H}$ (Fig. $1, C$ and $D$ ). Previous reports (Frics and Rothman, 1980) have already described the increased mobility of endo H-susceptible glycoproteins on SDS-PAGE after an enzyme treatment (Fig. 1, $A$ and $D$ ). Fetuin, a glycoprotein with endo $\mathrm{H}$-resistant, complex $\mathrm{N}$-linked oligosaccharides (Spiro, 1964), did not demonstrate increased electrophoretic mobility (Fig. 1, $E$ and $F$ ) or a loss of Con A binding (Fig. $1, G$ and $H$ ) after endo $\mathrm{H}$ digestion. These results emphasize that the enzymatic action of endo $\mathrm{H}$ on glycoproteins can be determined using lectin binding studies.

\section{The cold block technique}

A cold block of the sciatic nerve in rats (12 animals) to interrupt axonal transport in combination with postembedding lectin cytochemistry was used to study the carbohydrate composition of glycoproteins undergoing anterograde axonal transport. The cold block procedure used in this study was similar to a method reported to cause physical trauma to the nerve (Tsukita and Ishikawa, 1979). In our experiments, one vibratome slice from each segment of a nerve used for cytochemical analysis was processed for electron microscopy. In all sections of control or experimental nerves, no sign of physical damage was found at the light- or electron-microscopic levels.

\section{Electron microscopy}

Ultrastructural differences between control and experimental nerves were observed in axons directly proximal to the chilled silver wire $\left(0-5^{\circ} \mathrm{C}\right)$ compared to axons directly proximal to a silver wire held at ambient temperature (Fig. 2, $A$ and $B$ ). Axons 
in the control nerve or outside the cold block area in experimental nerves had the typical ultrastructural composition of axons in the PNS (Fig. 2A), including a sparse distribution of membrane vesicles typically located in the peripheral axoplasm. A prominent increase in smooth membrane vesicles in the peripheral axoplasm was a general feature of axons directly proximal to the cold block (Fig. $2 B$ ). At the cold block some axonal profiles not showing a noticeable increase in smooth membrane vesicles in the internodal segment did have a large increase in membrane profiles at the node of Ranvier (not shown). This increase was over and above the usual amount of membrane organelles observed at the nodes of axons in control nerves.

\section{Glycosidase enzyme digestion and lectin cytochemistry}

A cold block of the sciatic nerve for $6 \mathrm{hr}$ with the incorporation of the proximal warming device resulted in increased axonal Con A binding sites directly proximal $(0.5 \mathrm{~mm}$ or less) to the site where the chilled silver wire was applied around the nerve (Fig. $3 A$ ). In regions of the nerve outside the cold block area or in the control nerve (opposite sciatic nerve) directly proximal to a silver wire maintained at ambient temperature, the axons had very light, if any, Con A-peroxidase reaction product (Fig. $3 B$ ). Tissue sections taken directly proximal to the cold block and incubated with Con A plus the hapten sugar $(0.2 \mathrm{M} \alpha$-methyl-D-mannoside) did not exhibit peroxidase reaction product (Fig. $3 A^{\prime}$ ). This demonstrated that the staining pattern was specific for Con A binding sites, and also showed that the reaction product did not result from endogenous peroxidase activity in the tissue. A protease digestion ( $0.1 \%$ in PBS) of etched tissue sections for $3 \mathrm{hr}$ at $37^{\circ} \mathrm{C}$ prior to lectin cytochemical staining removed the Con A binding sites in the axons (not shown), suggesting that most of the lectin binding sites were glycoprotein carbohydrates.

Endo $\mathrm{H}$ digestion of etched sections from a cold block nerve resulted in a substantial decrease in Con A peroxidase labeling in axons (Fig. 3, $E$ and $F$ ). Enzyme control experiments using etched sections from the same area of the cold block nerve as endo $\mathrm{H}$-treated sections showed a heavy peroxidase reaction product after Con A labeling (Fig. $3, C$ and $D$ ). The enzyme control experiments also demonstrated that an incubation at low $\mathrm{pH}(\mathrm{pH} \mathrm{5.6)}$ for an extended period of time $(20 \mathrm{hr})$ did not destroy or alter the lectin binding sites. The biochemical characterization of axonal Con A binding sites, visualized by cytochemical labeling, as endo $\mathrm{H}$-sensitive showed that a population of the transported carbohydrates was $\mathrm{N}$-linked polymannose and/or hybrid oligosaccharides.

Axons in the cold block nerve that were heavily labeled with Con A (Fig. 3A) were also found to be highly fluorescent after WGA-FITC labeling (Fig. $3 G$ ). Hapten control experiments with $0.2 \mathrm{M}$ GlcNAc and WGA-FITC resulted in no observable fluorescence (Fig. $G^{\prime}$ ) in sections taken from the same area as in Figure $3 G$. Neuraminidase digestion of etched tissue sections taken from the same tissue block as the Con A and WGA studies showed a decrease, but not a total loss, of WGA binding sites in some axons (Fig. $3 H$ ).

The partial loss of WGA-FITC labeling in axons after a neuraminidase enzyme digestion indicated that only a certain portion of these lectin binding sites were dependent on a terminal NANA residue. Previously, we have shown that the cell surface of neurons and the neuropil of the cerebellar deep nucleus contain WGA binding sites that are completely removed by neuraminidase digestion (Hart and Wood, 1985; Wood et al., 1981).

To investigate further the nature of the neuraminidase-resistant WGA binding sites, sWGA lectin cytochemistry was performed on control and cold block nerves. Axons in control nerves or in experimental nerves outside the cold block area did not contain sWGA-FITC binding sites observable at the light-microscopic level (Fig. 3I). Axons at the cold block site

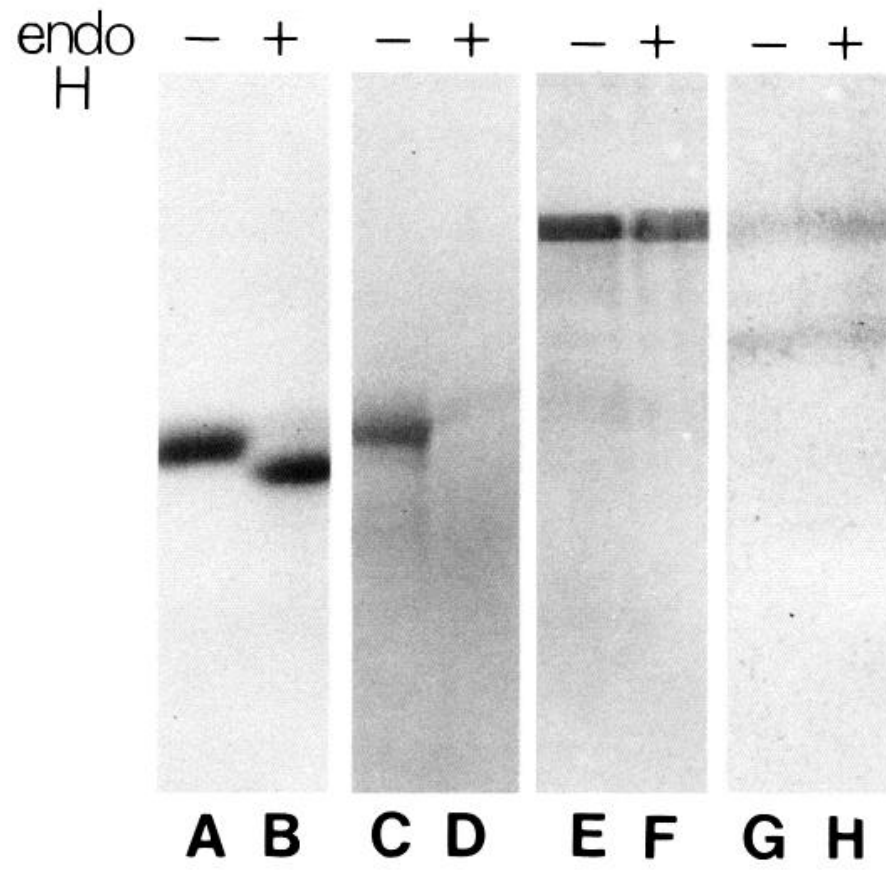

Figure 1. SDS-PAGE and lectin labeling of endo H-treated glycoproteins. Ovalbumin $(0.5 \mathrm{mg})$ and fetuin $(0.5 \mathrm{mg})$ were each incubated with endo $\mathrm{H}(7.5 \mathrm{mU})$ in $0.1 \mathrm{~m}$ citrate-phosphate buffer $(\mathrm{pH} \mathrm{5.6)}$ for $20 \mathrm{hr}$ at $37^{\circ} \mathrm{C}$. Control experiments, run in parallel with the enzyme digestions, incubated the glycoproteins in buffer minus the enzyme for $20 \mathrm{hr}$ at $37^{\circ} \mathrm{C}$. Aliquots of the incubation mixtures were diluted in a sample buffer and electrophoresed using a $12 \%$ acrylamide gel. Gels stained with Coomassie blue showed that ovalbumin migrated further into the gel after endo $\mathrm{H}$ digestion (lane $B$ ) compared to an incubation in buffer alone (lane $A$ ). Lectin staining of the gel by the method of Wood and Sarinana (1975) showed that the Con A binding sites of ovalbumin (lane $C$ ) were removed by an endo $\mathrm{H}$ digestion (lane $D$ ). Coomassie blue staining of gels also showed that the migration of fetuin (lane $E$ ) was not affected by endo $\mathrm{H}$ digestion (lane $F$ ). Also, nitrocellulose transblots of fetuin gels showed that the Con A labeling of fetuin (lane $G$ ) was not inhibited by an endo $\mathrm{H}$ digestion (lane $H$ ).

did contain a prominent sWGA-FITC fluorescence (Fig. $3 J$ ). Since sWGA does not bind to glycoconjugates containing terminal NANA residues but does bind to glycoconjugates containing GlcNAc (Monsigny et al., 1980; Wood et al., 1984), these results complement the results showing a significant pool of neuraminidase-resistant WGA binding sites proximal to the cold block. It should be noted that, although virtually all cell surface WGA binding sites are neuraminidase-sensitive, the presence of NANA is not an absolute requirement for WGA binding to glycoconjugates. The intense neuraminidase-insensitive binding of WGA to opsin (Wood et al., 1984) is a good example, since this photoreceptor glycoprotein contains terminal GlcNAc residues and no sialic acid. By analogy to opsin, it is tempting to speculate that the pool of sWGA-positive and neuraminidase-insensitive/WGA-positive binding sites proximal to the cold block may be composed of terminal GlcNAc residues on glycoconjugates, but we have no independent evidence for this. These results do, however, continue to indicate that the cytochemical properties of a pool of intracellular axonally transported glycoconjugates differ from the bulk of the plasma membrane glycoconjugates, and this difference must be explained. Hapten control experiments with $0.2 \mathrm{M}$ GlcNAc and sWGA-FITC displayed no observable fluorescence (not shown).

\section{Discussion}

The axonal transport blocks used in this study provided a means of increasing the population of anterogradely transported glycoproteins at a focal site along the sciatic nerve. This was an 

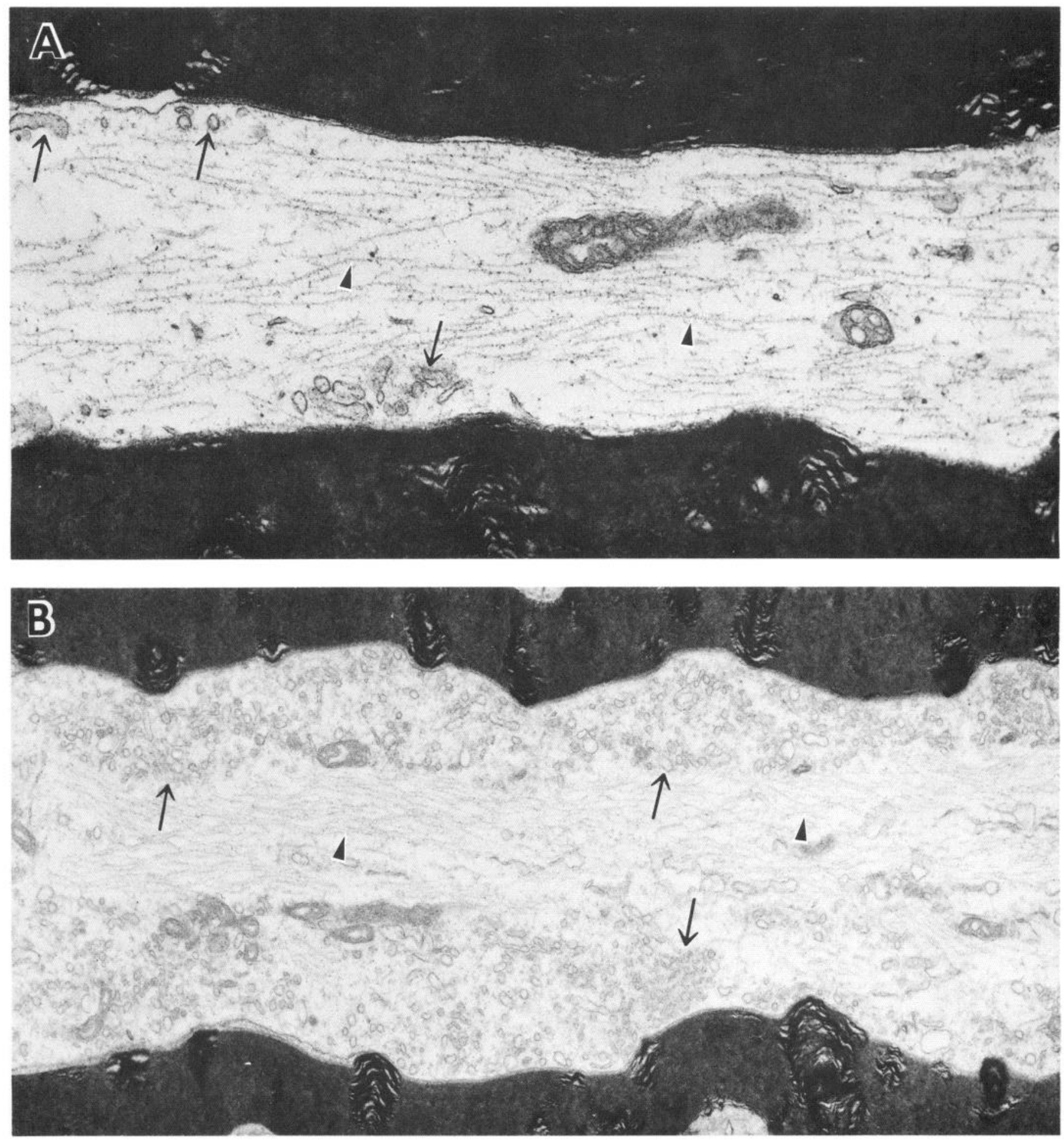

Figure 2. Electron microscopy of axons in control and cold block nerves. A, Axons in control nerves, shown in this micrograph, or axons outside the cold block area in experimental nerves had a normal distribution of smooth membrane vesicles (arrows) in the peripheral axoplasm and an abundance of neurofilaments (arrowheads) $(\times 37,000)$. B. Axons inside the cold block area of an experimental nerve had a large accumulation of smooth membrane vesicles (arrows) in the peripheral axoplasm and a clumping of neurofilaments (arrowheads) in the center of the axon. $\times 18,150$.

essential prerequisite for studying the transported glycoprotein carbohydrates because axonal profiles in either control nerves or experimental nerves outside the cold block area exhibited very few lectin binding sites at the light-microscopic level. More importantly, the cold block allowed for a selective increase of binding sites moving in the anterograde direction.
Electron-microscopic examination of the sciatic nerve directly proximal to the cold block showed an increased number of smooth membrane vesicles in axons when compared to the control nerve or in the experimental nerve greater than $0.5 \mathrm{~mm}$ proximal to the cold block. Previous reports using cold block techniques (Ellisman and Lindsey, 1983; Tsukita and Ishikawa, 

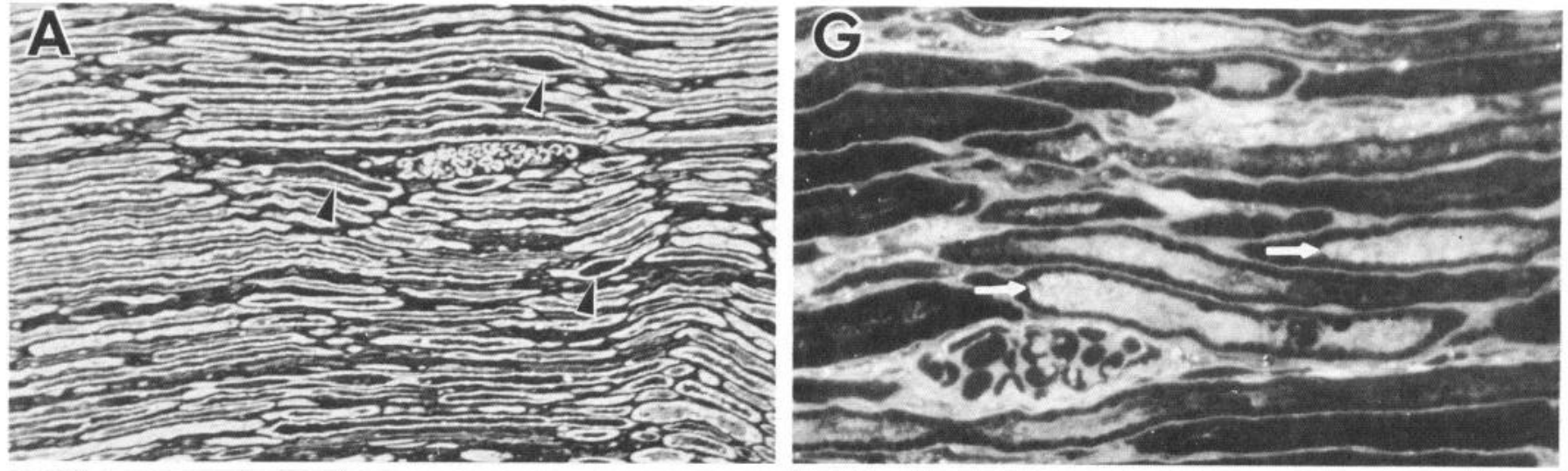

$\mathbf{A}^{\prime}$
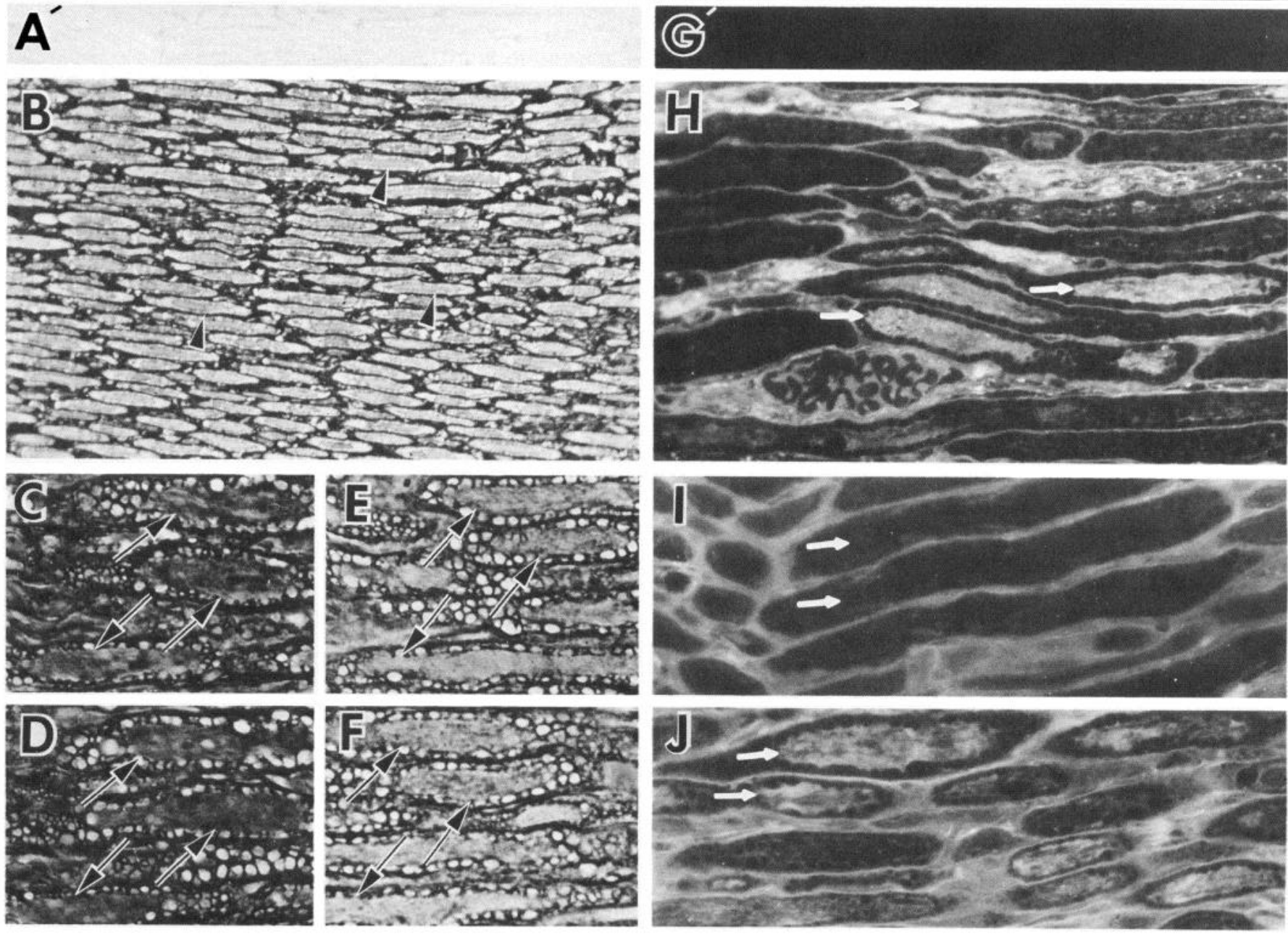

Figure 3. Postembedding lectin cytochemistry and glycosidase digestions of the sciatic nerve. A, Con A-peroxidase labeling of the sciatic nerve at a site directly proximal to the cold block. Axons contained a large amount of Con A binding sites (arrowheads) as shown by the peroxidase reaction product. $\left(A^{\prime}\right)$ Hapten control experiment of Con A binding at the same site in the nerve as in $A$. $B$, Con A-peroxidase labeling of the control sciatic nerve at a site directly proximal to a silver wire maintained at ambient temperature. Very light, if any, Con A-peroxidase label can be seen in the axons (arrowheads). $C$ and $D$, Sections of the cold block nerve were incubated in buffer minus endo $\mathrm{H}$. Axons (arrows) were heavily labeled with Con A-peroxidase. $E$ and $F$, Endo $\mathrm{H}$ digestion of sections from the same area of the cold block nerve $(C, D)$ show that the enzyme treatment removes a large population of the Con A binding sites in axons (arrows). $G$, WGA-FITC labeling of sections from the same cold block nerve in $A$. Axons that showed a high content of Con A binding sites were also heavily labeled with WGA-FITC (arrows). $G^{\prime}$, Hapten control experiment with WGA-FITC and $0.2 \mathrm{M} \mathrm{N}$-acetylglucosamine. $H$, Neuraminidase digestion of sections from a cold block sciatic nerve labeled with WGA-FITC. This section was taken from the same area as in $G$. The enzyme treatment reduced, but did not completely remove, the fluorescent label in axons (arrows). I, Axons (arrows) outside the cold block area or in control nerves were not labeled with sWGA-FITC. $J$, Axons (arrows) at the cold block site showed fluorescent labeling with sWGA-FITC. $A$ and $B, \times 250 ; C-F, \times 665 ; G$ and $H, \times 565 ; I$ and $J, \times 665$. 
1979,1980 ), or a slight compression of single myelinated axons (Smith, 1981) to interrupt fast axonal transport have also described an accumulation of smooth membrane vesicles in axons directly proximal to a transport block. The reports cited above in combination with electron-microscopic autoradiographic and subcellular fractionation studies have repeatedly indicated that the majority of axonally transported glycoproteins are membrane bound (see Ambron and Schwartz, 1979; Elam, 1979, for references).

The use of endo $\mathrm{H}$ digestions to characterize axonally transported Con $\mathrm{A}$ binding sites showed that a significant population of these glycoprotein carbohydrates consists of polymannose or hybrid oligosaccharides. This is of interest since very little is known about the actual oligosaccharide content of axonally transported glycoconjugates (e.g., Cole and Elam, 1983). It is known, however, that exogenously applied lectins are axonally transported in both the antero- and retrograde direction (Harper et al., 1980; LaVail et al., 1983; Nennesmo and Kristensson, 1981; Ruda and Coulter, 1982), and that transported radiolabeled glycoproteins bind to lectin affinity columns (Cole and Elam, 1981; Karlsson, 1979, 1980). We have previously shown that Con A binding sites in smooth membrane cisternae of dendrites and presynaptic terminals are endo $\mathrm{H}$-susceptible and that Con $A$ binding sites of the neuronal cell surface are endo H-resistant (Hart and Wood, 1984).

The neuraminidase-resistant WGA binding sites and the sWGA labeling of the axons directly proximal to the cold block demonstrated a distinct population of axonally transported carbohydrates that were not terminally glycosylated with NANA. In the CNS we have observed presynaptic terminals that also contain a large intracellular population of neuraminidase-resistant WGA and sWGA binding sites (Hart and Wood, 1985). Conversely, almost all the WGA binding sites in the neuropil and in synaptic clefts are neuraminidase-susceptible (Hart and Wood, 1985; Wood et al., 1981). In this regard, biochemical analysis of nervous tissue indicates the presence of an unusually high number of unsubstituted galactose and GlcNAc residues in membrane-bound, $\mathrm{N}$-linked oligosaccharides (Krusius and Finne, 1977). Interestingly, all the results from our previous reports on glycosidase digestion-lectin cytochemical experiments and the results presented here suggest that, at least in neurons, the bulk of this pool of membrane oligosaccharides, not terminally glycosylated with NANA, is intracellular.

It has been reported that all proteins, including glycoproteins, destined for the axolemma or presynaptic terminal plasma membrane are translated in the RER, pass through the Golgi complex (the reported site of terminal glycosylation), and are associated with axonal and synaptic terminal smooth membrane systems during their transport (see Hammerschlag, 1984; Hammerschlag et al., 1982, for references). The differences between lectin binding sites on the cell surface of neurons compared to intracellular compartments in the axons, dendrites, and presynaptic terminals (sites that receive Golgi-processed glycoproteins) could indicate that some post-Golgi processing of glycoprotein carbohydrates may occur in neurons. Other explanations for this data, however, must be considered. Differences in the accessibility of the lectins and enzymes to intracellular compartments compared to the cell surface might be a factor in the differential lectin staining pattern. Another possibility is that the intracellular lectin binding sites in neuronal processes are not precursors of the cell surface. This study was designed to address these possibilities since most of the glycoproteins in axons are destined for the synaptic terminal cell surface (Elam, 1979) or the axolemma (Griffin et al., 1981), and we selectively increased and assayed this population by the axonal transport blocks. It is possible, however, that a specific population of the glycoproteins undergoing axonal transport are not destined for the axolemma or synaptic terminal cell surface, because they remain as intracellular glycoproteins in a membrane system subserving the transport of cell surface macromolecules. Under these circumstances a relatively high proportion of the Con A-positive/endo H-susceptible and WGA-positive/neuraminidase-resistant lectin binding sites would be intracellular glycoconjugates that are not destined for the neuronal cell surface. The axonally transported glycoconjugates destined for the cell surface could have complex oligosaccharides that are relatively Con A-negative/endo H-resistant and WGA-positive/neuraminidase-susceptible.

The possibility that lectin binding sites in intracellular domains and on the cell surface are differentially accessible to enzymes and lectins is not likely since endo H-susceptible sites are intracellular (this report; and Hart and Wood, 1984) and neuraminidase-susceptible sites are both intra- and extracellular (this report; and Hart and Wood, 1985; Wood et al., 1981). It is certainly possible that synaptic terminals in the CNS have a different carbohydrate composition than the neuromuscular presynaptic endings of the sciatic nerve axons studied here. It is known that neuromuscular junctions stain with Con $A$ and WGA (Sanes and Cheney, 1982), hut carbohydrate analysis of the lectin binding sites by glycosidase enzyme digestion was not reported.

The results in this report and our previous lectin cytochemical studies have led us to consider the possibility that postGolgi modification of glycoprotein carbohydrates may occur in nerve cell processes. The reports of glycosyltransferase activity in synaptosomal fractions derived from brain homogenates (Den et al., 1975; Dutton et al., 1973; Goodrum et al., 1979; Preti et al., 1980; Rostas et al., 1981; White and Dawson, 1981) and the observation that ${ }^{3} \mathrm{H}-\mathrm{N}$-acetylgalactosamine injected directly into the L2 axons of Aplysia is incorporated into glycoproteins undergoing axonal transport (Ambron and Treistman, 1977) support the concept of oligosaccharide modification in postGolgi compartments. Posttranslational modification of axonally transported protein (Tedeschi and Wilson, 1983), unequal turnover of the peptide backbone compared to sugar moieties of transported glycoproteins (Goodrum and Morrell, 1984), and the identification of sialidase and sialoglycoprotein substrates in rat brain synaptic junctions (Cruz and Gurd, 1983) are also consistent with the possibility of a self-contained mechanism at the synaptic terminal capable of expressing a variety of cell surface properties. We do not suggest, however, that all glycoprotein carbohydrates destined for the synaptic cell surface are processed by a post-Golgi mechanism. One approach to studying the possibility of post-Golgi mechanisms would be to perform the endo $\mathrm{H}$ and neuraminidase-linked lectin studies on transported radiolabeled glycoconjugates isolated from nerve and presynaptic terminals and separated by lectin-affinity chromatography and PAGE. These experiments are in progress. If a process is identified in which certain glycoprotein oligosaccharides are modified at or close to the point of their insertion into the synaptic plasma membrane, a mechanism would exist whereby these molecules could be important in the maintenance and plasticity of synaptic contacts.

\section{References}

Ambron, R. T., and S. N. Treistman (1977) Glycoproteins are modified in the axon of R2, the giant neuron of Aplysia california, after intra-axonal injection of ${ }^{3} \mathrm{H}-\mathrm{N}$-acetylgalactosamine. Brain Res. 121: 287-309.

Ambron, R. T., and J. H. Schwartz (1979) Regional aspects of neuronal glycoprotein and glycolipid synthesis. In Complex Carbohydrates of Nervous Tissue, R. U. Margolis and R. K. Margolis, eds., pp. 269290, Plenum, New York.

Bhavanandan, V.P., and A. W. Katlic (1979) The interaction of wheat germ agglutinin with sialoglycoconjugates. J. Biol. Chem. 254:40004008 .

Cassidy, J. T., G. W. Jourdian, and S. Roseman (1965) The sialic 
acids. VI. Purification and properties of sialidase from Clostridium perfringens. J. Biol. Chem. 240: 3501-3506.

Cole, G. J., and J. S. Elam (1981) Axonal transport of glycoproteins in regenerating olfactory nerve: Enhanced glycopeptide concanavalin A-binding. Brain Res. 222: 437-441

Cole, G. J., and J. S. Elam (1983) Characterization of axonally transported glycoproteins in regenerating garfish olfactory nerve. J. Neurochem. 41: 691-702.

Cruz, T. F., and J. W. Gurd (1983) Identification of intrinsic sialidase and sialoglycoprotein substrates in rat brain synaptic junctions. $J$. Neurochem. 40: 1599-1604.

Den, H., B. Kaufman, E. J. McGuire, and S. Roseman (1975) The sialic acids. XVIII. Subcellular distribution of seven glycosyntransferases in embryonic chicken brain. J. Biol. Chem. 250: 739-746.

Dutton, G. R., P. Haywood, and S. H. Barondes (1973) $\left[{ }^{14} \mathrm{C}\right]$ Glucosamine incorporation into specific products in the nerve ending fraction in vivo and in vitro. Brain Res. 57: 397-408.

Elam, J. S. (1979) Axonal transport of complex carbohydrates. In Complex Carbohydrates of Nervous Tissue, R. U. Margolis and R. K. Margolis, eds., pp. 235-268, Plenum, New York.

Ellisman, M. H., and J. D. Lindsey (1983) The axoplasmic reticulum within myelinated axons is not transported rapidly. J. Neurocytol. 12: 393-411.

Fries, E., and J. E. Rothman (1980) Transport of vesicular stomatitis virus glycoprotein in a cell-free extract. Proc. Natl. Acad. Sci. USA 77: $3870-3874$.

Goodrum, J. F., and P. Morell (1984) Analysis of the apparent biphasic axonal transport kinetics of fucosylated glycoproteins. J. Neurosci. 4: $1830-1839$.

Goodrum, J. F., H. B. Bosmann, and R. Tanaka (1979) Glycoprotein galactosyltransferase activity in synaptic junctional complexes isolated from rat forebrain. Neurochem. Res. 4: 331-337.

Grafstein, B., and D. S. Forman (1980) Intracellular transport in neurons. Physiol. Rev. 60: 1167-1283.

Griffin, J. W., D. L. Price, D. B. Drachman, and J. Morris (1981) Incorporation of axonally transported glycoproteins into the axolemma during nerve regeneration. J. Cell Biol. 88: 205-214.

Hammerschlag, R. (1984) How do neuronal proteins know where they are going? Speculations on the role of molecular address markers. Dev. Neurosci. 6: 2-17.

Hammerschlag, R., and G. C. Stone (1982) Membrane delivery by fast axonal transport. Trends Neurosci. 5: 12-15.

Hammerschlag, R., G. C. Stone, F. A. Bolen, J. D. Lindsey, and M. H. Ellisman (1982) Evidence that all newly synthesized proteins destined for fast axonal transport pass through the Golgi apparatus. J. Cell Biol. 93: 575-586.

Hanson, M. (1978) A new method to study fast axonal transport in vivo. Brain Res. 153: 121-126.

Harper, C. G., J. O. Gonatas, S. Stieber, and N. K. Gonatas (1980) In vivo uptake of WGA-HRP conjugates into neuronal GERL and lysosome. Brain Res. 188: 465-472.

Hart, C. E., and J. G. Wood (1983) Axonal transport of lectin binding sites. J. Cell Biol. 97: 241a (abstr.).

Hart, C. E., and J. G. Wood (1984) Endoglycosidase H digestion of neuronal intracellular lecting binding sites. J. Neurosci. 4: 1589-1597.

Hart, C. E., and J. G. Wood (1985) A comparative study of intracellular lectin binding sites of neurons in culture with neurons in situ. J. Comp. Neurol. 239: 155-162.

Karlsson, J. O. (1979) Proteins of axonal transport: Interaction of rapidly transported proteins with lectins. J. Neurochem. 32: 491494.

Karlsson, J. O. (1980) Proteins of rapid axonal transport: Polypeptides interacting with the lectin from Lens culinaris. J. Neurochem. 34 . $1184-1190$.

Kobata, A. (1979) Use of endo- and exoglycosidases for structural studies of glycoproteins. Anal. Biochem. 100: 1-14.

Krusius, T., and J. Finne (1977) Structural features of tissue glyco- proteins: Fractionation and methylation analysis of glycopeptides derived from rat brain. Eur. J. Biochem. 78: 369-379.

Laemmli, V.D. (1970) Cleavage of structural proteins during assembly of the head of bacteriophage T4. Nature 227: 680-685.

Lane, B. P., and D. L. Europa (1965) Differential staining of ultrathin sections of epon-embedded tissues for light microscopy. J. Histochem. Cytochem. 13: 579-582.

LaVail, J. H., I. K. Sugino, and D. M. McDonald (1983) Localization of axonally transported ${ }^{125}$ I-wheat germ agglutinin beneath the plasma membrane of chick retinal ganglion cells. J. Cell Biol. 96: 373381.

Lowry, O. H., N. J. Rosebrough, A. L. Farr, and R. J. Randall (1951) Protein measurement with the Folin phenol reagent. J. Biol. Chem. 193: 265-275.

Millonig, G. (1961) Advantages of a phosphate buffer of $\mathrm{OsO}_{4}$ solutions in fixation. J. Appl. Phys. 32: 1637 (abstr.).

Monsigny, M., A.-C. Roche, C. Sene, R. Maget-Dana, and F. Delmotte (1980) Sugar-lectin interactions: How does wheat germ agglutinin bind sialoglycoproteins? Eur. J. Biochem. 104: 147-153.

Nennesmo, I., and K. Kirstensson (1981) Somatopetal axonal transport of fluorescent lectins: Distribution pattern and cytophotometric quantification in mousc pcriphcral neurons. Neurosci. Lett. 27: 243248.

Preti, A., A. Fiorilli, A. Lombardo, L. Caimi, and G. Tettamanti (1980) Occurrence of sialytransferase activity in synaptosomal membranes prepared from calf brain cortex. J. Neurochem. 35: 281-296.

Rostas, J. A. P., W. N. Leung, and P. L. Jeffrey (1981) Glycosyltransferase activities in chicken brain synaptic junctions. Neurosci. Lett. 24: 155-160.

Ruda, M., and J. D. Coulter (1982) Axonal and transneuronal transport of wheat germ agglutinin demonstrated by immunocytochemistry. Brain Res. 249: 237-246.

Sanes, J. R., and J. M. Cheney (1982) Lectin binding reveals a synapsespecific carbohydrate in skeletal muscle. Nature 300:646-647.

Smith, R. S. (1981) The short term accumulation of axonally transported organelles in the region of localized lesions of single myelinated axons. J. Neurocytol. 9: 39-65.

Spiro, R. G. (1964) Periodate oxidation of the glycoprotein fetuin. J. Biol, Chem. 239: 567-573.

Tedeschi, B., and D. L. Wilson (1983) Modification of a rapidly transported protein in regenerating nerve. J. Neurosci. 3: 1728-1734

Towbin, H. G., T. Stahelin, and J. Gordon (1979) Electrophoretic transfer of proteins from polyacrylamide gels to nitrocellulose sheets. Procedure and some applications. Proc. Natl. Acad. Sci. USA 76 : $4350-4354$.

Tsukita, S., and H. Ishikawa (1979) Morphological evidence for the involvement of smooth endoplasmic reticulum in axonal transport. Brain Res. 174: 315-318.

Tsukita, S., and H. Ishikawa (1980) The movement of membranous organelles in axons: Electron microscopic identification of anterogradely and retrogradely transported organelles. J. Cell Biol. 84: 513530.

White, C. J., and J. M. Dawson (1981) Fucose incorporation and identification of fucosylglycoproteins in synaptosomes. J. Neurochem. 37: 1155-1163.

Wood, J. G., and B. J. McLaughlin (1976) Cytochemical studies of lectin binding sites in smooth membrane cisternae of rat brain. Brain Res. 118: 15-26.

Wood, J. G., and F. O. Sarinana (1975) The staining of sciatic nerve glycoproteins on polyacrylamide gels with Concanavalin A-peroxidase. Anal. Biochem. 69: 329-322.

Wood, J. G., B. J. McLaughlin, and R. P. Barber (1974) The visualization of concanavalin A binding sites in Purkinje cell somata and dendrites of rat cerebellum. J. Cell Biol. 63: 541-549.

Wood, J. G., F. I. Byrd, and J. W. Gurd (1981) Lectin cytochemistry of carbohydrates on cell membranes of rat cerebellum. J. Neurocytol. 10: $149-159$. 